\title{
Patterns of inspiratory muscle shortening during hypoxia and hypercapnia in dogs
}

\author{
M. Suzuki, S. Suzuki, T. Akahori, A. Miyashita, T. Yoshioka, M. Sato, T. Okubo
}

\begin{abstract}
Patterns of inspiratory muscle shortening during hypoxia and hypercapnia in dogs. $M$. Suzuki, S. Suzuki, T. Akahori, A. Miyashita, T. Yoshioka, M. Sato, T. Okubo. @ERS Journals Ltd 1997.

ABSTRACT: The shortening of parasternal intercostal muscles (Para) and crural (Cru) and costal diaphragms (Cos) are not precisely understood. We therefore examined shortening patterns of these inspiratory muscles by using chronically implanted sonomicrometers in dogs.

To avoid acute effects of surgery, measurements were performed 3 weeks after implanting the sonomicrometers. Patterns of length changes of Para, Cru, and Cos were measured during hypoxia and hypercapnia under two levels of anaesthesia.

Respiratory length change $(\Delta \mathbf{L})$ was assessed as a percentage change relative to the resting length at functional residual capacity (LFRC). Peak tidal shortening was defined as the maximal change from LFRC $(\triangle \mathrm{L} / \mathrm{LFRC})$. Under light anesthesia, the $\Delta \mathrm{L} / \mathrm{LFRC}$ was the same among the three muscle groups at all tidal volumes $(V T)$. Under deep anaesthesia, the $\Delta \mathrm{L} / \mathrm{LFRC}$ both of Cru and Cos exceeded that of Para. Under light anaesthesia, the maximal shortening velocity $((\Delta \mathrm{L} / \mathrm{LFRC}) / \Delta t)$ of $\mathrm{Cru}$ was greater than that of Para. Under deep anaesthesia, the $(\Delta \mathrm{L} / \mathrm{LFRC}) / \Delta t$ of Para was exceeded by that both of Cru and Cos. Furthermore, the $(\Delta \mathrm{L} / \mathrm{LFRC}) / \Delta t$ of each inspiratory muscle was greater during hypoxia than during hypercapnia at equal volume.

We conclude that: 1$)$ the contribution of the diaphragm to ventilation increases during deep anaesthesia; 2 ) the muscle shortening velocity during hypoxia or hypercapnia is lower in parasternal intercostal muscles than in the diaphragm; and 3) there is no difference in the shortening pattern between crural and costal diaphragms. Eur Respir J 1997; 10: 430-436.
\end{abstract}

The First Department of Internal Medicine, Yokohama City University School of Medicine, Yokohama, Japan

\section{Correspondence: S. Suzuki}

The First Department of Internal Medicine Yokohama City University School of Medicine

3-9, Fukuura

Kanazawa-ku

Yokohama 236

Japan

Keywords: Anaesthesia diaphragm

hypoxia and hypercapnia parasternal intercostal muscle

Received: March 71995

Accepted after revision January 311996
Among the respiratory muscles of the chest wall, the intercostal muscles and the diaphragm act as the major respiratory muscles for breathing. Several reports have suggested that parasternal intercostal muscles (Para) are the primary muscles responsible for expanding the upper rib cage during inspiration $[1,2]$. The diaphragm is anatomically divided into two muscles, the crural (Cru) and costal parts (Cos) [3]. In animals, the activities of the inspiratory muscles have been studied mainly by electromyography (EMG) $[4,5]$. These studies have demonstrated that the intercostal muscles, Cru, and Cos are activated differently. On the other hand, studies on the mechanical activities of the inspiratory muscles have focused primarily on their overall movements, i.e. the rib cage and abdominal movements, by inductive plethysmography [6], or the volume-pressure relationships of the rib cage and the diaphragm-abdomen [7]. These studies have suggested that the contribution of the rib cage and diaphragm to ventilation is altered by the magnitude of ventilation or by anaesthesia.

Recently, direct measurements of the changes in the lengths of the respiratory muscles have been reported by the use of sonomicrometry $[8,9]$. This technique allows an accurate measurement of any change in the length of respiratory muscles in vivo. However, EASTON and co-workers [10] found that there was a transient inhibition of diaphragmatic shortening after the upper abdominal surgery required for the implantation of the devices. This suggests that the chronic implantation of sonomicrometric transducers would provide more reliable results than measurements obtained shortly after implantation. Recently, EASTON and co-workers [11] reported that Cru and Cos responded differently to hypercapnia in dogs chronically-implanted with sonomicrometric transducers.

Since hypoxia primarily stimulates peripheral chemoreceptors and affects the pattern of breathing or of phrenic discharge differently from hypercapnia [7, 12], we hypothesized that inspiratory muscles, such as Para, Cru and Cos, behave differently during isocapnic hypoxia and hypercapnia. In addition, we reasoned that the motion of the Para may be affected by the depth of general anaesthesia more than the diaphragm, because the rib cage movements have been shown to decrease by inducing general anaesthesia [6]. To test this hypothesis, the shortening patterns of each inspiratory muscle were studied during hypoxia and hypercapnia under two different levels of anaesthesia. In this study, the mechanical activities of anaesthetized canine Para, Cru and Cos were measured by using chronically-implanted sonomicrometric transducers. 


\section{Material and methods}

\section{Surgical procedure}

A total of 10 dogs (weighing 9-15 kg) were anaesthetized with an intravenous injection of thiopental sodium $\left(25 \mathrm{mg} \cdot \mathrm{kg}^{-1}\right)$, and were maintained by its supplemental infusion. Sonomicrometric transducers were implanted in the diaphragm as reported previously [13]. A pair of sonomicrometric transducers (Murata Manufacturing Co., Kyoto, Japan), 2.5-3.0 $\mathrm{mm}$ in diameter and covered with epoxy resin, was implanted between the peritoneum and the muscle fibres. Each pair of the transducers was inserted into the central portion of the medial Cru and the dorsal Cos. The transducers were oriented along the direction of the muscle fibres and were fixed 10-18 $\mathrm{mm}$ apart. The implantation of the transducers onto Para was based on the method described by DeCRAMER et al. [8]. The Para of the right 4th intercostal space was exposed. A pair of transducers was inserted into small pockets opened between muscle fibres $8-16 \mathrm{~mm}$ apart, and was fixed in place in an orientation along the muscle fibres. The muscle fascia was closed with sutures. The lead-in wires were placed in subcutaneous pockets.

\section{Measurements}

Three weeks after implanting the transducers, the animals were anaesthetized with an intravenous injection of thiopental sodium $\left(15 \mathrm{mg} \cdot \mathrm{kg}^{-1}\right)$. They were placed supine, intubated with a cuffed endotracheal tube that was attached via a heated pneumotachograph (Fleisch No. 1, Lausanne, Switzerland) to a two-way non-rebreathing valve (Model 1400; Hans-Rudolph, MO, USA). Tidal airflow was measured from the pressure drop across the pneumotachograph (MP-45; Validyne, CA, USA) and electrically integrated to give tidal volume. A catheter was inserted into the femoral artery to obtain samples for blood gas analysis (Model micro 13; Instrumentation Laboratory, MA, USA). Expired gas was monitored by a gas analyser (Model 1H21A; NEC San-ei, Tokyo, Japan). Body temperature was maintained at $38^{\circ} \mathrm{C}$ with a heating pad. The lead-in wires from the sonomicrometric transducers were connected directly to amplifiers (Model 4105; NEC San-ei). The sonomicrometry signals, tidal airflow and volume were recorded on an 8-channel strip recorder (Rectigraph 8K; NEC San-ei). The data were also processed with an analogue-to-digital (A-D) converting circuit (Analog Pro II; Canopus, Kobe, Japan) and a microcomputer (PC-9801 VM-4; NEC, Tokyo).

\section{Protocol}

Measurements were initially performed under light anaesthesia, and the same protocol was repeated under deep anaesthesia. Each level of anaesthesia was maintained by intravenous administration of supplemental thiopental. In each level of anaesthesia, fractional endtidal carbon dioxide $\left(F \mathrm{ET}, \mathrm{CO}_{2}\right)$ and arterial carbon dioxide tension $\left(\mathrm{Pa}_{\mathrm{a}} \mathrm{CO}_{2}\right)$ during air breathing were kept constant.
Throughout light anaesthesia, the corneal reflex was kept intact. After taking control measurements during air breathing, the inspiratory circuit was switched to a Douglas bag (capacity $200 \mathrm{~L}$ ) containing a hypoxic or hypercapnic gas mixture. Two levels of oxygen concentration were used for the hypoxic mixtures, i.e. $15 \% \mathrm{O}_{2} /$ $85 \%$ nitrogen, and $10 \% \mathrm{O}_{2} / 90 \%$ nitrogen ("15 and $10 \%$ $\mathrm{O}_{2}{ }^{\prime \prime)}$. During hypoxia, $10 \% \mathrm{CO}_{2}$ gas balanced with nitrogen was added to the inspiratory circuit to maintain isocapnia. Two levels of $\mathrm{CO}_{2}$ concentration were used for the hypercapnic mixtures, i.e. $7 \% \mathrm{CO}_{2}$ or $10 \% \mathrm{CO}_{2}$ with $30 \% \mathrm{O}_{2}$ and $63 \%$ or $60 \%$ nitrogen, respectively. The inhalation of these four gas mixtures $\left(15 \% \mathrm{O}_{2}, 10 \% \mathrm{O}_{2}\right.$, $7 \% \mathrm{CO}_{2}$ and $10 \% \mathrm{CO}_{2}$ ) was performed in random order. Animals inhaled each gas mixture for $5 \mathrm{~min}$. Data obtained from the last $1 \mathrm{~min}$ were reported. After each measurement, the animals were allowed $15 \mathrm{~min}$ to recover. A control measurement was then repeated, followed by measurements with another gas mixture. When the measurements under light anesthesia had been completed, thiopental sodium was added intravenously until the corneal reflex was completely suppressed $(10 \sim 15$ $\mathrm{mg} \cdot \mathrm{kg}^{-1}$ ), and the same protocol as under light anaesthesia was repeated. Finally, the animals were killed with an overdose of aneasthetics, and the condition of the inspiratory muscles at the implantation sites was examined macroscopically and microscopically.

\section{Data analysis}

Respiratory length changes $(\Delta \mathrm{L})$ in Para, Cru and Cos were assessed as a percentage change relative to the resting length at functional residual capacity (LFRC). Peak tidal shortening was defined as the maximal change from LFRC $(\triangle \mathrm{L} / \mathrm{LFRC} \%)$. The maximal shortening velocity of an inspiratory muscle $\left((\Delta \mathrm{L} / \mathrm{LFRC} \%) / \Delta t, \% \cdot \mathrm{s}^{-1}\right)$ was calculated from the steepest part of the slope of muscle length (L) against time during inspiration. The inspiratory time $(t \mathrm{I})$, expiratory time $(t \mathrm{E})$, total breath duration $(t$ tot $)(=t \mathrm{I}+t \mathrm{E})$ were determined from the $V \mathrm{~T}$ and airflow signals. The minute ventilation $\left(V^{\prime} \mathrm{E}\right)$, respiratory frequency $(f \mathrm{R})$, mean inspiratory flow $(V \mathrm{~T} / t \mathrm{I})$, and duty ratio $\left(t \mathrm{I} / t_{\text {tot }}\right)$ were also calculated. Data were averaged for the group of animals and are presented as the mean \pm SE.

\section{Statistics}

The effects of the level of anaesthesia on the hypercapnic or hypoxic ventilatory responses were tested by comparing the slopes of the response curves, i.e. $\Delta V$ 'E/ $\Delta P \mathrm{a}, \mathrm{CO}_{2}, \Delta V \mathrm{~T} / \Delta P \mathrm{a}, \mathrm{CO}_{2}$ and $\Delta f \mathrm{R} / \Delta P \mathrm{a}, \mathrm{CO}_{2}$. Statistical comparison between groups was made by using a two-way analysis of variance (ANOVA) with repeated measures on two or three factors, followed by a post-hoc comparison using the Newman-Keuls test, and baseline values were compared by paired t-test. A p-value less than 0.05 was considered significant.

\section{Results}

In each muscle group, sonomicrometry signals were recorded from seven pairs of transducers from 10 implanted 
dogs; three pairs in each muscle group failed to retain signals because of a loss of alignment during the postoperative period. An example of the respiratory changes in muscle length is shown in figure 1. Marked increases in peak tidal shortening $(\Delta \mathrm{L} / \mathrm{LFRC})$ and maximal shortening velocity $((\Delta \mathrm{L} / \mathrm{LFRC}) / \Delta t)$ were observed in each inspiratory muscle during $10 \% \mathrm{CO}_{2}$. The LFRC during quiet breathing was $9.9 \pm 1.3 \mathrm{~mm}$ in Para, $13.8 \pm 2.0 \mathrm{~mm}$ in Cru, and $14.0 \pm 1.3 \mathrm{~mm}$ in Cos. They showed a tendency to increase during hypercapnia but did not reach a significant difference. They were not affected by the level of anaesthesia.

During the measurements of quiet breathing, the $F$ ET, $\mathrm{CO}_{2}$ and $P \mathrm{a}, \mathrm{CO}_{2}$ were kept constant. The $F \mathrm{ET}, \mathrm{CO}_{2}$ was $5.1 \pm 0.1 \%$ under light anaesthesia and increased to $6.0 \pm 0.1 \%$ under deep anaesthesia $(\mathrm{p}<0.01)$. The $P \mathrm{a}, \mathrm{CO}_{2}$ was $5.1 \pm 0.1 \mathrm{kPa}$ during light anesthesia and reached $5.8 \pm 0.1 \mathrm{kPa}$ under deep anaesthesia $(\mathrm{p}<0.01)$. Under deep anaesthesia, the $V^{\prime} \mathrm{E}$ during quiet breathing decreased by $22.4 \%$ compared with that under light anaesthesia $(\mathrm{p}<0.01)$. During 15 and $10 \% \mathrm{O}_{2}$, the $\mathrm{Pa}_{\mathrm{a}} \mathrm{CO}_{2}$ remained at $4.7 \pm 0.2 \mathrm{kPa}$ under light anaesthesia and $5.3 \pm 0.3 \mathrm{kPa}$ under deep anaesthesia. During 7 or $10 \% \mathrm{CO}_{2}$, the arterial oxygen tension $\left(\mathrm{Pa}_{\mathrm{a}} \mathrm{O}_{2}\right)$ reached $25.7 \pm 2.3 \mathrm{kPa}$. During deep anaesthesia, the responses of $V^{\prime} \mathrm{E}$ and $f \mathrm{R}$ were decreased both in hypoxia and hypercapnia compared to light anaesthesia (all $\mathrm{p}<0.01$ by ANOVA) (fig. 2). However, the $V \mathrm{~T}$ was not affected by the level of anaesthesia in hypoxia or hypercapnia. Thus, during deep anaesthesia, the ventilatory response both to hypoxia and hypercapnia was suppressed compared to light anaesthesia, due mainly to a decreased response of respiratory rate.

Hypoxia affected respiratory timing to a greater extent than hypercapnia during deep anaesthesia, but not during light anaesthesia. The $\Delta f \mathrm{R} / \Delta V \mathrm{~T}$ obtained from the hypoxic runs was greater than that from the hypercapnic tests $(\mathrm{p}<0.05$ by ANOVA) (fig. 3a). However, the level of anaesthesia did not affect the $\Delta f \mathrm{R} / \Delta V \mathrm{~T}$. Both the $t \mathrm{I}$ and $t \mathrm{E}$ obtained from hypoxic runs had greater responses $(\Delta t \mathrm{I} / \Delta V \mathrm{~T}$ and $\Delta t \mathrm{E} / \Delta V \mathrm{~T})$ compared to those under hypercapnia $(\mathrm{p}<0.01$ and $\mathrm{p}<0.05$ by ANOVA, respectively)

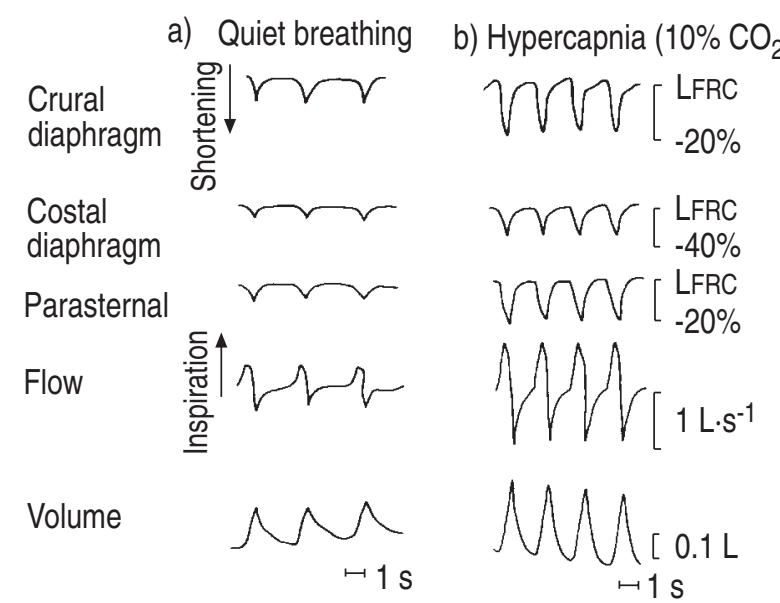

Fig. 1. - Representative tracings of the inspiratory muscle length changes during: a) quiet breathing; and b) hypercapnia, under light anaesthesia. Deflection of the baseline indicates muscle shortening. Note the marked increases in the length changes of the inspiratory muscles during hypercapnia. LFRC: inspiratory muscle length at functional residual capacity. (fig. 3b). In the $t \mathrm{E}$, deep anaesthesia had greater responses compared to light anesthesia ( $\mathrm{p}<0.05$ by ANOVA). In the relationship between $V \mathrm{~T} / t \mathrm{I}$ and $V \mathrm{~T}$ hypoxia had an increased response compared to hypercapnia $(\mathrm{p}<0.01$ by ANOVA), although the level of anaesthesia did not affect the response of $V \mathrm{~T} / t \mathrm{I}$ (fig. $3 \mathrm{c}$ ). When the duty ratio was

$$
\text { a) }
$$

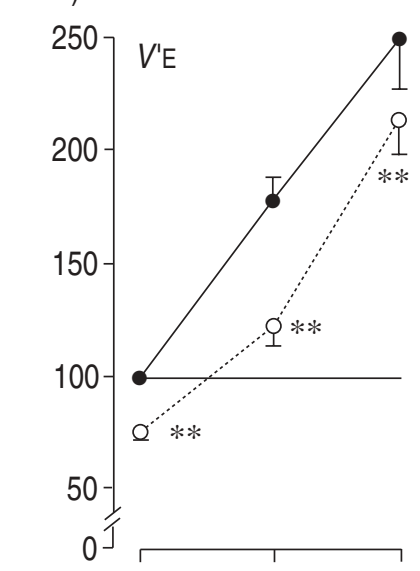

b)
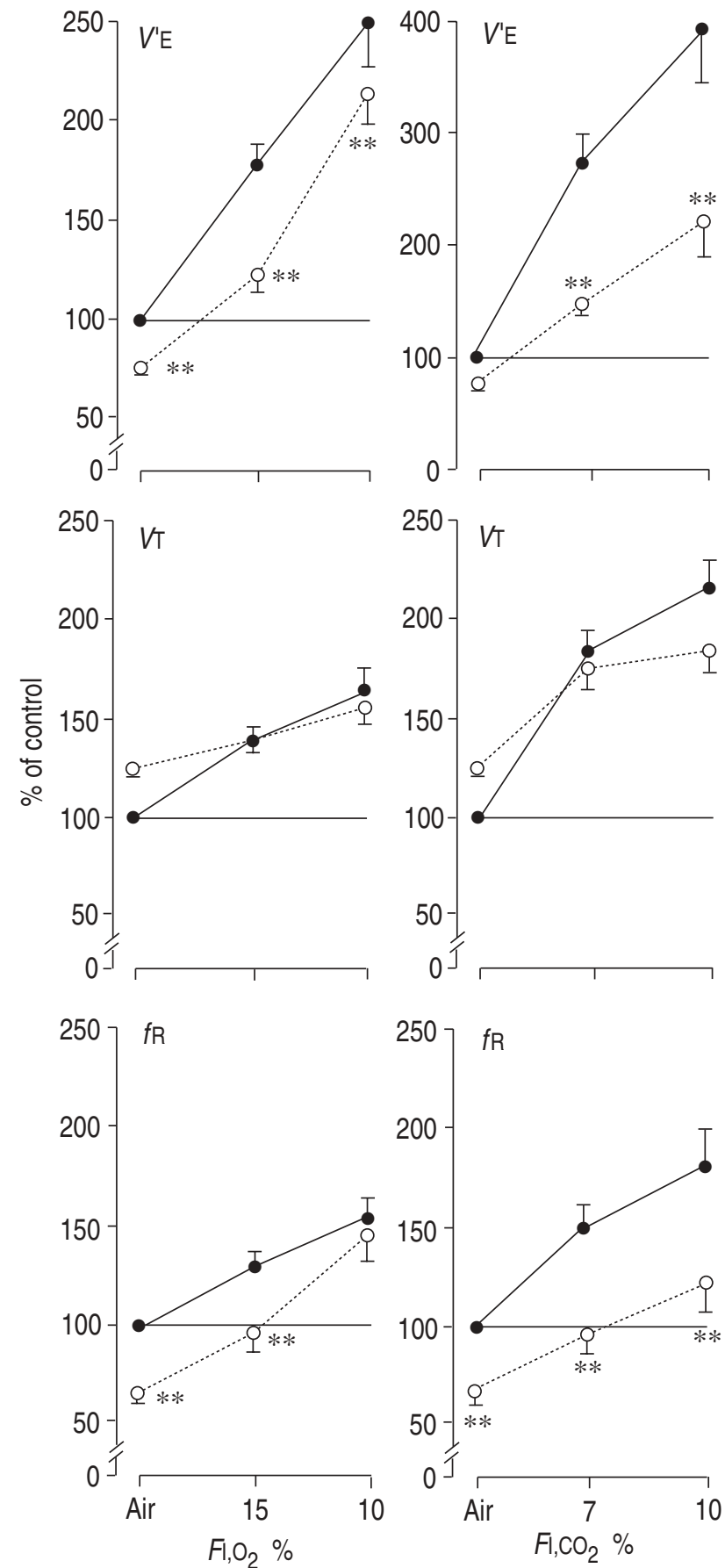

Fig. 2. - Effects of the level of anaesthesia on the ventilatory responses to: a) hypoxia; and b) hypercapnia. Values are presented as mean \pm SE $(\mathrm{n}=10)$. The responses of minute ventilation $\left(V^{\prime} \mathrm{E}\right)$ and respiratory frequency $(f \mathrm{R})$ to hypoxia or hypercapnia were decreased during deep anaesthesia $(\ldots . . . \cdots . .$.$) compared to light anaesthesia (-\bullet)($ all $\mathrm{p}<0.01$ by ANOVA), whilst the response of tidal volume $(V \mathrm{~T})$ to hypoxia or hypercapnia was not changed by the level of anaesthesia. $* *: p<0.01$, compared to light anaesthesia (Newman-Keuls test). 

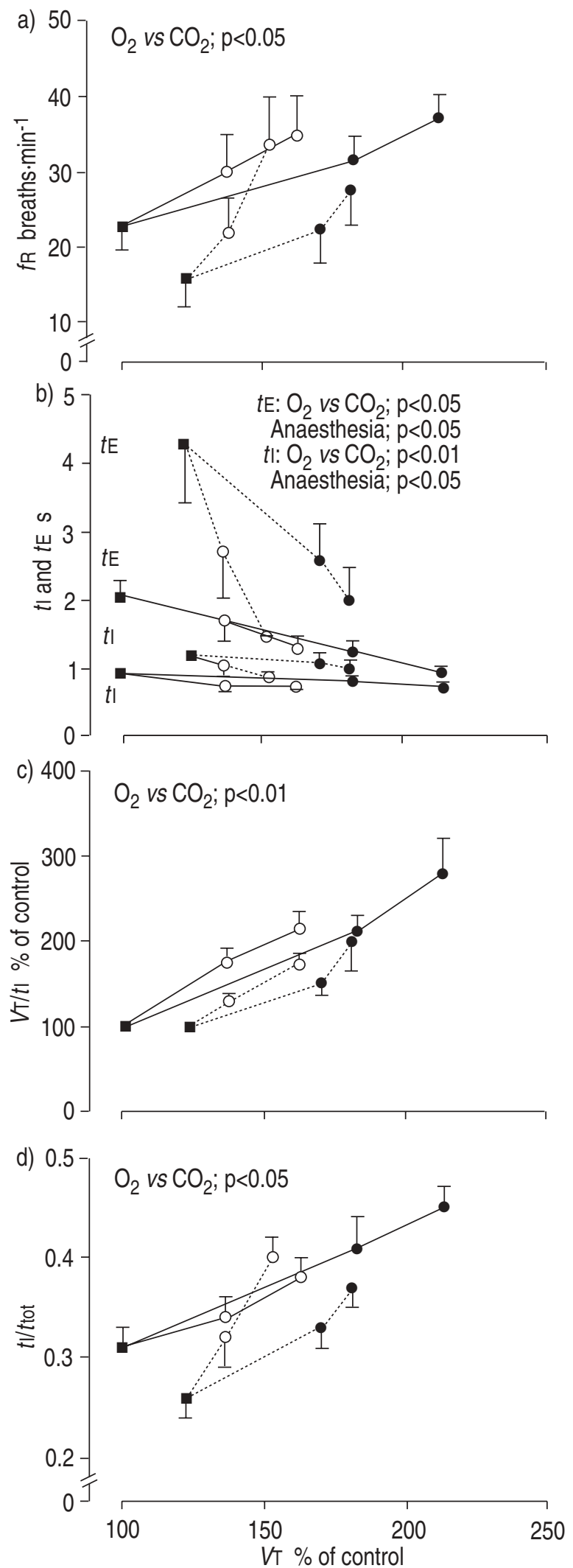

Fig. 3. - a) Relationship between the respiratory frequency $(f R)$ and tidal volume $(V \mathrm{~T})$. b) Inspiratory time $(t \mathrm{I})$ and expiratory time $(t \mathrm{E})$ plotted against $V$ T. c) The relationship between the mean inspiratory flow rate $(V \mathrm{~T} / t \mathrm{I})$ and $V \mathrm{~T}$. d) The duty ratio $(t \mathrm{I} / t$ tot $)$ plotted against $V \mathrm{~T}$. Values are presented as mean \pm SE $(n=10)$. The error bars for $V$ T are omitted. See the text for details. $t$ tot: total breath duration. __ : light anaesthesia; ......... deep anaesthesia; $\mathbf{\square}$ : air; $\bullet$ : hypercapnia; ○: hypoxia. plotted against $V$ T (fig. 3d), no difference was found between hypoxia and hypercapnia during light anaesthesia. During deep anaesthesia, however, hypoxia increased the duty ratio in proportion to $V \mathrm{~T}(\Delta[t \mathrm{I} / t$ tot $) / \Delta V \mathrm{~T})$ more than did hypercapnia $(\mathrm{p}<0.05$ by ANOVA).

The peak tidal shortening $(\triangle \mathrm{L} / \mathrm{LFRC})$ of each inspiratory muscle group was plotted against $\mathrm{Pa}, \mathrm{CO}_{2}$ or $\mathrm{Pa}, \mathrm{O}_{2}$ (fig. 4). In hypercapnia, deep anaesthesia suppressed the responses of the muscles $\left(\Delta(\Delta \mathrm{L} / \mathrm{LFRC}) / \Delta P \mathrm{a}, \mathrm{CO}_{2}\right)$ $(\mathrm{p}<0.01$ by ANOVA). The response of Para was less than the other two muscles $(\mathrm{p}<0.05$ by ANOVA). In hypoxia, however, the responses in $\triangle \mathrm{L} / \mathrm{LFRC}$ were not affected by the level of anaesthesia in any of the three muscle groups.

The relationships between the peak tidal shortening $(\Delta \mathrm{L} / \mathrm{LFRC})$ and $V \mathrm{~T}(\Delta \mathrm{L}-V \mathrm{~T}$ relationships $)$ and between the maximal shortening velocity $(\Delta \mathrm{L} / \mathrm{LFRC}) / \Delta t$ and $V \mathrm{~T}$
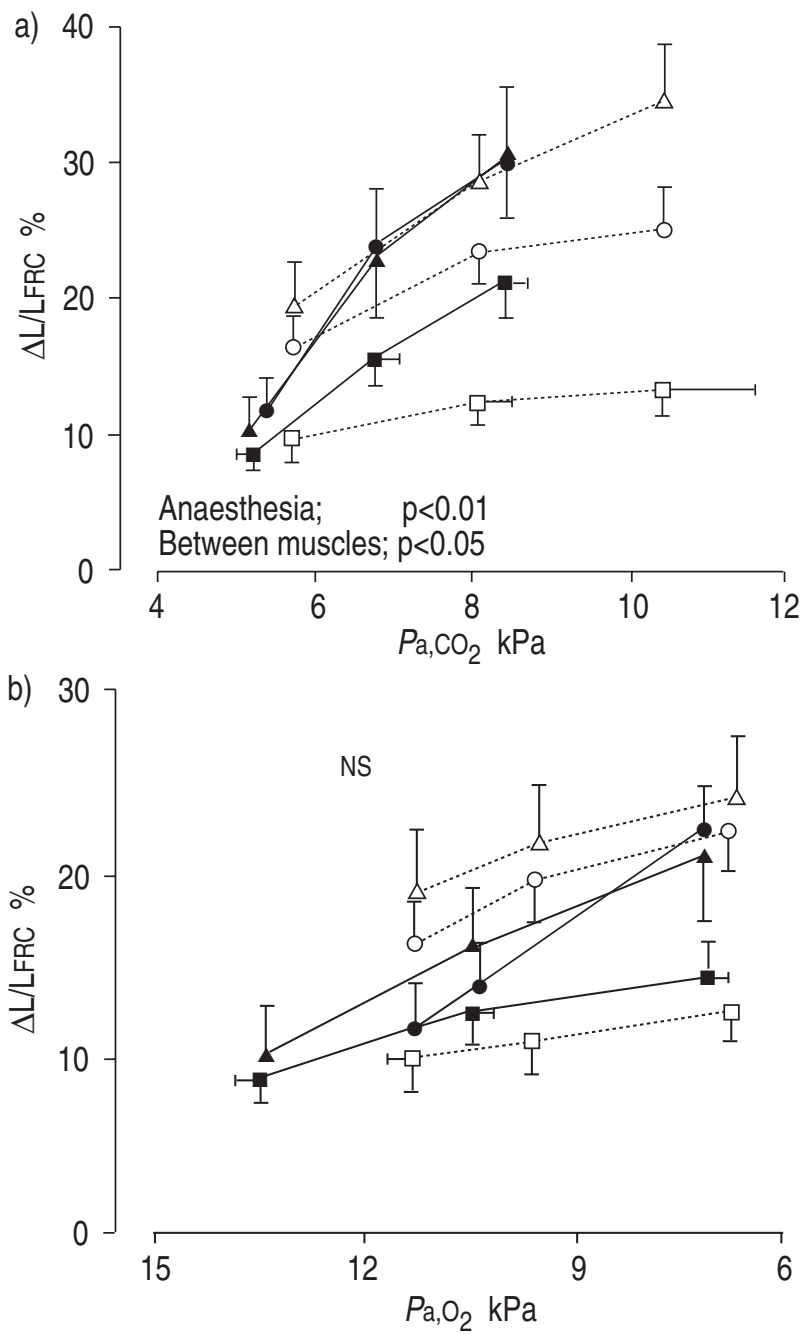

Fig. 4. - Peak tidal shortening of each muscle during: a) hypercapnia; and b) hypoxia. Values are presented as mean \pm SE $(n=7)$. In hypercapnia, the response of $\Delta(\Delta \mathrm{L} / \mathrm{LFRC}) / \Delta V \mathrm{~T}$ of each muscle decreased during deep anaesthesia compared to light anaesthesia $(\mathrm{p}<0.01$ by ANOVA $)$, and there was a significant difference in $\Delta(\Delta \mathrm{L} / \mathrm{LFRC}) / V_{\mathrm{T}}$ between the three muscles ( $\mathrm{p}<0.05$ by ANOVA). $\mathbf{\square}$ and $\square$ : parasternal intercostal muscles; $\Delta$ and $\Delta$ : crural diaphragm; $\bullet$ and $O$ : costal diaphragm;__ ; light anaesthesia; …… : piratory length change; LFRC: length at functional residual capacity; $\Delta V$ T: change in tidal volume; $\Delta \mathrm{L} / \mathrm{LFRC}$ : peak tidal shortening; $P_{\mathrm{a}, \mathrm{CO}_{2}}$ : arterial carbon dioxide tension; $\mathrm{Pa}_{\mathrm{a}, \mathrm{O}_{2}}$ : arterial oxygen tension; ANOVA: analysis of variance; NS: not significant. 
a)

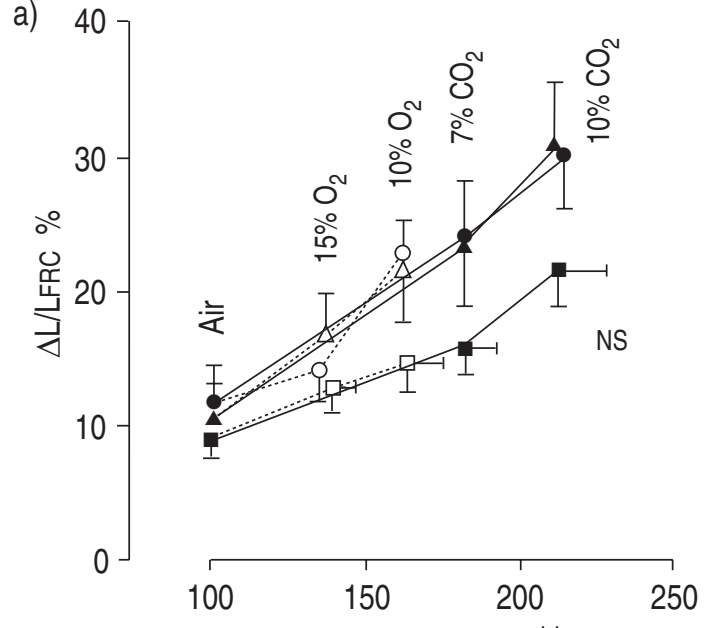

c)

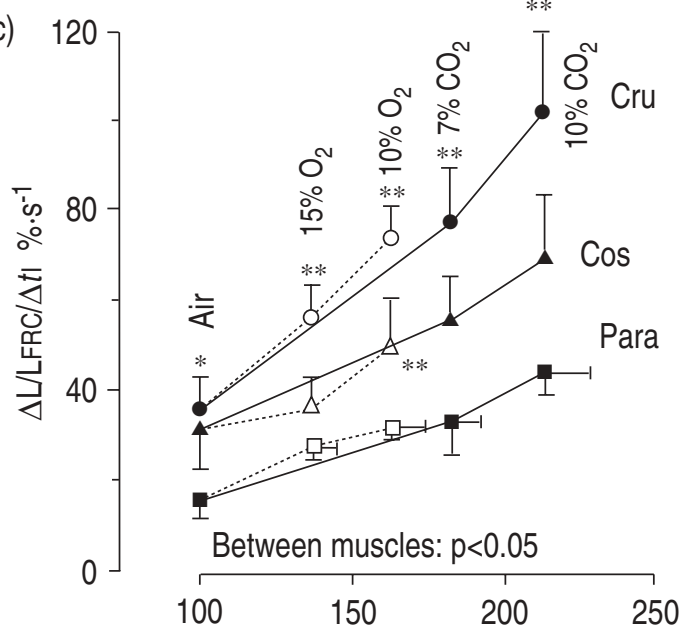

b)

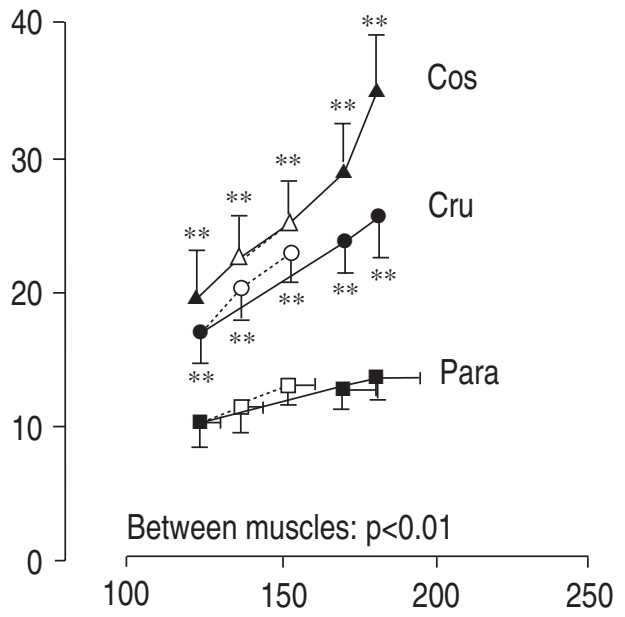

d)

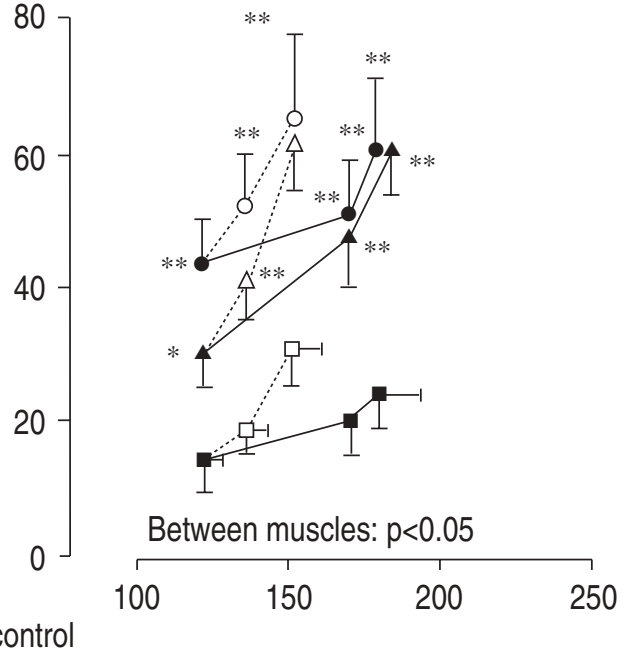

Fig. 5. - Relationships between $\triangle \mathrm{L} / \mathrm{LFRC}$ and $V_{\mathrm{T}}$ during hypoxia and hypercapnia: a) under light anaesthesia; and b) under deep anaesthesia. Relationship between $(\Delta \mathrm{L} / \mathrm{LFRC}) / \Delta t$ and $V \mathrm{~T})$ ); c) under light anaesthesia; and d) under deep anaesthesia. Values are presented as mean $\pm \mathrm{SE}(\mathrm{n}=7)$. Under deep anaesthesia, the $\Delta \mathrm{L} / \mathrm{LFRC}$ of the parasternal intercostal muscles (Para) was lower than that of the other two muscles $(\mathrm{p}<0.01$ by ANOVA) (b). The maximal shortening velocity $(\Delta \mathrm{L} / \mathrm{LFRC}) / \Delta t$, was different between nuscles during light anaesthesia ( $\mathrm{p}<0.05$ by ANOVA) (c). During deep anaesthesia, the $\Delta \mathrm{L} / \mathrm{LFRC} / \Delta t$ both of the crural diaphragm (Cru) and costal diaphragm (Cos) was greater than that of Para (p<0.05 by ANOVA) (d). The error bars for the $V \mathrm{~T}$ are presented in Para. *: $\mathrm{p}<0.05$ compared to Para by the Newman-Keuls test; **: $\mathrm{p}<0.01 \mathrm{compard}$ to Para._— : hypercapnia; ................ : hypoxia. ( $\triangle \mathrm{L} / \mathrm{LFRC}) / \Delta t$ : maximal shortening velocity. For further definitions see legend to figure 4 .

are presented in figure 5. Under light anaesthesia, the $\triangle \mathrm{L} / \mathrm{LFRC}$ of the three muscle groups was not different at any $V \mathrm{~T}$ (fig. 5a). However, under deep anaesthesia, the $\Delta \mathrm{L}-V \mathrm{~T}$ relationship was different in the three muscles ( $<0.01$ by ANOVA) (fig. $5 b$ ). The $\Delta$ L/LFRC of Cos was the greatest, while that of Para was the lowest and equalled about one-third of Cos. The crural $\Delta \mathrm{L}-V \mathrm{~T}$ relationship under deep anaesthesia was similar to that under light anaesthesia. No difference in $\triangle \mathrm{L} / \mathrm{LFRC}$ was found between the Cru and Cos or between hypoxia and hypercapnia. Thus, the $\Delta \mathrm{L} / \mathrm{LFRC}$ both of the Cru and Cos exceeded that of Para both during hypoxia and hypercapnia (Para vs Cru and Para vs Cos, all $\mathrm{p}<0.01$ at any $V \mathrm{~T})$. The maximal shortening velocity $(\Delta \mathrm{L} / \mathrm{LFRC}) /$ $\Delta t$ of Cru during light anaesthesia exceeded that of Para $\left(\mathrm{p}<0.01\right.$ at 10 and $15 \% \mathrm{O}_{2}$, and 7 and $15 \% \mathrm{CO}_{2}$ ) (fig. $5 \mathrm{c})$. Under deep anaesthesia, the $(\Delta \mathrm{L} / \mathrm{LFRC}) / \Delta t$ of Para was lower than those both of Cru and Cos (Para vs Cru; $\mathrm{p}<0.01$ at 7 and $10 \% \mathrm{O}_{2}$, and 10 and $15 \% \mathrm{CO}_{2}$; Para vs $\mathrm{Cos}, \mathrm{p}<0.01$ at all $V \mathrm{~T})$. Furthermore, the $\Delta((\Delta \mathrm{L} / \mathrm{LFRC}) / \Delta t)$
$/ \Delta V \mathrm{~T}$ obtained during hypoxia exceeded that during hypercapnia in all three muscle groups ( $<<0.05$ by ANOVA). Thus, during deep anaesthesia, the relationship between $(\Delta \mathrm{L} / \mathrm{LFRC}) / \Delta t$ and $V \mathrm{~T}$ was different not only in each inspiratory muscle group but also between hypoxia and hypercapnia.

At autopsy, the diaphragm had no fibrous connection to other abdominal tissues. The muscle fibres between the sonomicrometric transducers appeared to be intact. On microscopic examination, a slightly fibrotic capsule was found surrounding all the transducers, but no inflammatory cells or fibrotic changes extending into the intervening muscles were observed. In Para, a gross examination revealed no evidence of traction or torsion that could affect its length change. The muscle fibres between the implantation sites appeared to be intact. Microscopically, similar findings to those found in the diaphragm were observed, i.e. thin fibrotic capsules $(1-1.5 \mathrm{~mm})$ encasing the sonomicrometric transducers, and intact muscle fibres between them. 


\section{Discussion}

The main findings of this study were as follows: 1) the maximal muscle shortening velocity during hypoxia and hypercapnia is lower in Para than in the diaphragm; and 2) the level of anaesthesia affects the contribution of various inspiratory muscles to ventilation during hypoxia and hypercapnia.

\section{Critique on methodology}

Measurements were made in the animals 3 weeks after the implantation of the sonomicrometric transducers, because EASTON and co-workers [10] had reported that diaphragmatic shortening had normalized by the 10th day after implantation. We have previously reported that the $\triangle \mathrm{L} /$ LFRC of Cru and Cos obtained during supramaximal phrenic nerve stimulation reached a plateau on the 14th day after the implantation of the sonomicrometers, and then remained constant for the next 4 weeks [13]. Furthermore, the magnitudes of the shortening were similar to those observed shortly after implantation [13]. Therefore, it is likely that the muscles remain intact after the chronic implantation of the sonomicrometers.

The $\Delta \mathrm{L} / \mathrm{LFRC}$ of the Para observed during quiet breathing was $8.3 \%$ under light anesthesia and $10 \%$ under deep anaesthesia. These figures are similar to the previously reported values obtained from anaesthetized dogs shortly after the implantation of the sonomicrometers [14-16], suggesting that the effect of chronic implantation of the sonomicrometers on the contractility of the Para was minimal.

Recent studies using electromyography (EMG) of the inspiratory muscles showed that the onset of CRU activation preceded that of Para and Cos $[4,11]$. Since we did not perform EMG on the inspiratory muscles, it is difficult to estimate the passive portion of shortening or elongation. According to DARIAN et al. [4] and EASTON et al. [11], the EMG activities of Cru, Cos and Para were observed throughout the inspiration, except for the onset of inspiration in Cos and Para. We obtained only a peak tidal shortening $(\Delta \mathrm{L})$, which occurs near a maximum activity of EMG [11]. Therefore, we believe that the increases and decreases in the amount of muscle shortening of the three muscles could be explained by changes in muscle activation.

\section{Ventilatory response}

In dogs, barbiturate has been shown to depress the ventilatory response both to hypercapnia and hypoxia [17]. In this study, the ventilatory response to hypoxia and hypercapnia was suppressed in different ways by anaesthesia (fig. 2). The hypoxic ventilatory response is primarily affected by the peripheral chemoreceptor activity, while the hypercapnic response is affected by central chemoreceptors [4]. This difference between the mechanism of the hypoxic and hypercapnic response may account for their different sensitivity to anaesthesia. There was a clear difference in the pattern of hypoxic and hypercapnic breathing under deep anaesthesia (fig. 3). This is similar to the findings of GARCIA and
Cherniack [18] in dogs anaesthetized with pentobarbital. However, we found no significant difference in the pattern of breathing between hypoxia and hypercapnia during light anaesthesia. Our results suggest that the magnitude of the difference in the pattern of breathing between hypoxia and hypercapnia depends on the level of anaesthesia.

\section{Peak tidal shortening}

The peak tidal shortening of each inspiratory muscle group was not different during hypoxia and hypercapnia under light anaesthesia (fig. 5a). However, under deep anaesthesia, the peak tidal shortening both of Cos and Cru was greater than that of Para (fig. 5b), suggesting an altered contribution of the diaphragm and Para to ventilation. Tusiewicz et al. [6] have compared the chest wall motions of awake and halothane-anaesthetized humans by inductive plethysmography. They reported an increased contribution of the diaphragm during anaesthesia, and concluded that this phenomenon was a result of diminished activity of Para because its EMG decreased during anaesthesia. This may suggest that the phrenic motor neuron pool may be more resistant to depression than that of the intercostal muscles. Why these neuron pools have different sensitivities remains unclear.

We found no difference between the $\Delta \mathrm{L} / \mathrm{LFRC}$ of Cru and Cos during quiet or stimulated breathing under either level of anaesthesia. In contrast, Newman et al. [9] and ROAD et al. [19] reported a greater crural shortening compared to COS when they made their measurements shortly after the sonomicrometers were implanted. However, SpRung et al. [20] have found that by measuring the length changes between chronically-implanted markers under biplane videofluorography, the $\Delta \mathrm{L} / \mathrm{LFRC}$ of the medial Cos exceeded that of Cru. The greater crural shortening observed in the previous reports may be secondary to the implantation sites of the sonomicrometers. DARIAN et al. [4] have shown that in anaesthetized dogs during hypoxia, the EMG activity of Cru and Para increased preferentially compared to Cos. EASTON and co-workers [11] showed that peak tidal shortening of Cru and Cos did not differ in awake dogs chronically-implanted with sonomicrometers, but shortening of Cru exceeded that of $\mathrm{Cos}$ in earliest inspiration. These may suggest that the neural activation of Cos is different from Cru or Para. In the present study, anaesthesia had different effects on the shortening of Cru and Cos in response to hypercapnia (fig. 4). This may be explained by a difference in the sensitivities of the motor neuron pools of these muscles to anaesthesia. In addition, there may be different neural activation mechanisms between the Cru and Cos.

\section{Maximal shortening velocity}

During light anaesthesia, we found a range of $(\Delta \mathrm{L} /$ LFRC)/ $\Delta t$ among the inspiratory muscles. The $(\Delta \mathrm{L} / \mathrm{LFRC}) /$ $\Delta t$ of Cru exceeded that of Para, whereas their $\Delta \mathrm{L} / \mathrm{LFRC}$ were similar (fig. 5a), suggesting a different shortening pattern between Cru and Para during light anaesthesia. The $(\Delta \mathrm{L} / \mathrm{LFRC}) / \Delta t$ of an inspiratory muscle at a certain neural drive may be affected by several mechanical factors. The force generating ability of Para was reportedly 
less than Cos with low frequency $(10-20 \mathrm{~Hz})$ stimulation, because of their different twitch characteristics [21]. This suggests that length-tension properties may be different among the muscles. With respect to muscle action, De Troyer and co-workers [22] have demonstrated that Cru displaced the abdomen alone, whereas Cos displaced the abdomen and the lower rib cage. It has also been shown that Para expands the upper rib cage by displacing the sternum [23]. Moreover, in the supine position, the abdominal load applied to Cru is greater than that to Cos [19]. This suggests that the relationship between neural drive and mechanical output is quite different among Para, Cru and Cos. One or more of these factors may account for the differences in the $\Delta \mathrm{L} / \mathrm{LFRC} / \Delta t$ between the diaphragm and Para.

In the present study, we demonstrated some differences in the shortening patterns between the parasternal intercostal muscles and crural diaphragm and between the parasternal intercostal muscles and costal diaphragm. However, their shortening patterns were obviously affected by the level of anaesthesia. The breathing pattern during hypoxia and hypercapnia was different from that during deep anaesthesia. The contribution of the different inspiratory muscles to ventilation was altered during deep anaesthesia. Therefore, data from anaesthetized animals require careful interpretation.

\section{References}

1. De Troyer A, Farkas GA. Linkage between parasternals and external intercostals during resting breathing. J Appl Physiol 1990; 69: 509-516.

2. De Troyer A. Inspiratory elevation of the ribs in the dog: primary role of the parasternals. J Appl Physiol 1991; 70: 1447-1455.

3. De Troyer A, Sampson M, Sigrist S, Macklem PT. The diaphragm: two muscles. Science 1981; 213: 237-238.

4. Darian GB, DiMarco AF, Kelsen SG, Supinski GS, Gottfried SB. Effects of progressive hypoxia on parasternal, costal and crural diaphragm activation. J Appl Physiol 1989; 66: 2579-2584.

5. Oyer LM, Knuth SL, Ward DK, Bartlett D. Patterns of neural and muscular electrical activity in costal and crural portions of the diaphragm. $J$ Appl Physiol 1989; 66: 2092-2100.

6. Tusiewicz K, Bryan AC, Froese AB. Contributions of changing rib cage/diaphragm interactions to the ventilatory depression of halothane anesthesia. Anesthesiology 1977; 47: 327-337.

7. Grimby G, Goldman M, Mead J. Respiratory muscle action inferred from rib cage and abdominal V-P partitioning. J Appl Physiol 1976; 41: 739-751.
8. Decramer M, De Troyer A. Respiratory changes in parasternal intercostal length. $J$ Appl Physiol: Respirat Environ Exercise Physiol 1984; 57: 1254-1260.

9. Newman SL, Road J, Bellemare F, Clozel JP, Lavigne CM, Grassino A. Respiratory muscle length measured by sonomicrometry. J Appl Physiol: Respirat Environ Exercise Physiol 1984; 56: 753-764.

10. Easton PA, Fitting J-W, Arnoux R, Guerraty A, Grassino AE. Recovery of diaphragm function after laparotomy and chronic sonomicrometer implantation. J Appl Physiol 1989; 66: 613-621.

11. Easton PA, Fitting JW, Arnoux R, Guerraty A, Grassino AE. Costal and crural diaphragm function during $\mathrm{CO}_{2}$ rebreathing in awake dogs. J Appl Physiol 1993; 74: 1406-1418.

12. Ledlie JF, Kelsen SG, Cherniack NS, Fishman AP. Effects of hypercapnia and hypoxia on phrenic nerve activity and respiratory timing. $J$ Appl Physiol: Respirat Environ Exercise Physiol 1981; 51: 732-738.

13. Miyashita A, Suzuki S, Suzuki M, et al. Effect of thyroid hormone on in vivo contractility of the canine diaphragm. Am Rev Respir Dis 1992; 145: 1456-1462.

14. Van Lunteren E, Cherniack NS. Electrical and mechanical activity of respiratory muscles during hypercapnia. J Appl Physiol 1986; 61: 719-727.

15. De Troyer A. The electromechanical response of canine inspiratory intercostal muscles to increased resistance: the cranial rib cage. J Physiol (Lond) 1992; 451: 445-461.

16. De Troyer A, Farkas G. Mechanics of the parasternal intercostals in prone dogs: statics and dynamics. $J$ Appl Physiol 1993; 74: 2757-2762.

17. Hirshman CA, McCullough RE, Cohen PJ, Weil JV. Hypoxic ventilatory drive in dogs during thiopental, ketamine, or pentobarbital anesthesia. Anesthesiology 1975; 43: 628-634.

18. Garcia A, Cherniack NS. Integrated phrenic activity in hypercapnia and hypoxia. Anesthesiology 1967; 28: 1029-1035

19. Road JD, Newman SL, Grassino A. Diaphragm length and breathing pattern changes during hypoxia and hypercapnia. Respir Physiol 1986; 65: 39-53.

20. Sprung J, Deschamps C, Hubmayr RD, Walters BJ, Rodarte JR. In vivo regional diaphragm function in dogs. J Appl Physiol 1989; 67: 655-662.

21. Farkas GA, Decramer M, Rochester DF, De Troyer A. Contractile properties of intercostal muscles and their functional significance. J Appl Physiol 1985; 59: 528-535.

22. De Troyer A, Sampson M, Sigrist S, Macklem PT. Action of costal and crural parts of the diaphragm on the rib cage in dog. J Appl Physiol: Respirat Environ Exercise Physiol 1982; 53: 30-39.

23. De Troyer A, Kelly D. Chest wall mechanics in dogs with acute diaphragm paralysis. J Appl Physiol: Respirat Environ Exercise Physiol 1982; 53: 373-379. 